\title{
Analytical Formulas for Radio Paths in Spherically Stratified Ionospheres
}

\author{
E. Woyk (Chvojkova)
}

\section{Contribution From the Astronomical Institute Czechoslovak Academy of Sciences, Praha 2}

\author{
(Received September 4, 1964; revised November 5, 1964)
}

\begin{abstract}
Analytical solutions are developed for tracing radio rays through a spherically stratified ionospheric layer. The electron density profile is represented by a series of parabolic sections, each section being bounded by levels of maxima or minima of electron density and by the middle values between these extremes.

With this model, relatively simple functions are obtained which give the length of the radius vector in terms of these levels. For example, it is shown that, under certain conditions, the ray path is a simple ellipse or a quasi-ellipse. The cases of reflection and penetration are considered for waves originating on the earth's surface and for waves incident from outside the ionosphere.
\end{abstract}

\section{Introduction}

The need for an analytical formula capable of expressing the path of a radio wave in the ionosphere was raised at the meeting on Oblique Sounding of the Ionosphere [IQSY Notes, 1963] held at Lindau, Germany, in May 1963. This led to the derivation of equations which express the following kinds of radio wave propagation: (a) penetration of the layer, (b) oscillation along a circular path, and (c) reflection. Although the formulas refer to propagation in the absence of the earth's magnetic field, they may be applied also if a slowly varying magnetic field is present.

\section{Propagation in Perfectly Spherical Ionized Layers}

A "perfectly spherical" layer is assumed to be one in which the levels of constant electron density $(N)$ are concentric spheres. Each sphere is defined by its radius of curvature $(r)$ (fig. la). The variation of electron density from level to level is quite arbitrary; it must, however, remain the same in the whole region investigated [Woyk, 1964].

For waves propagated in spherically ionized layers, there are two fundamental formulas defining the ray path: Snell's Law,

$r n \sin i=$ constant $=r_{m} \sin i_{c}=r_{t}=n r_{t}^{(n)}$

$$
=r_{r} n_{r}=r_{E} \sin i_{E}
$$
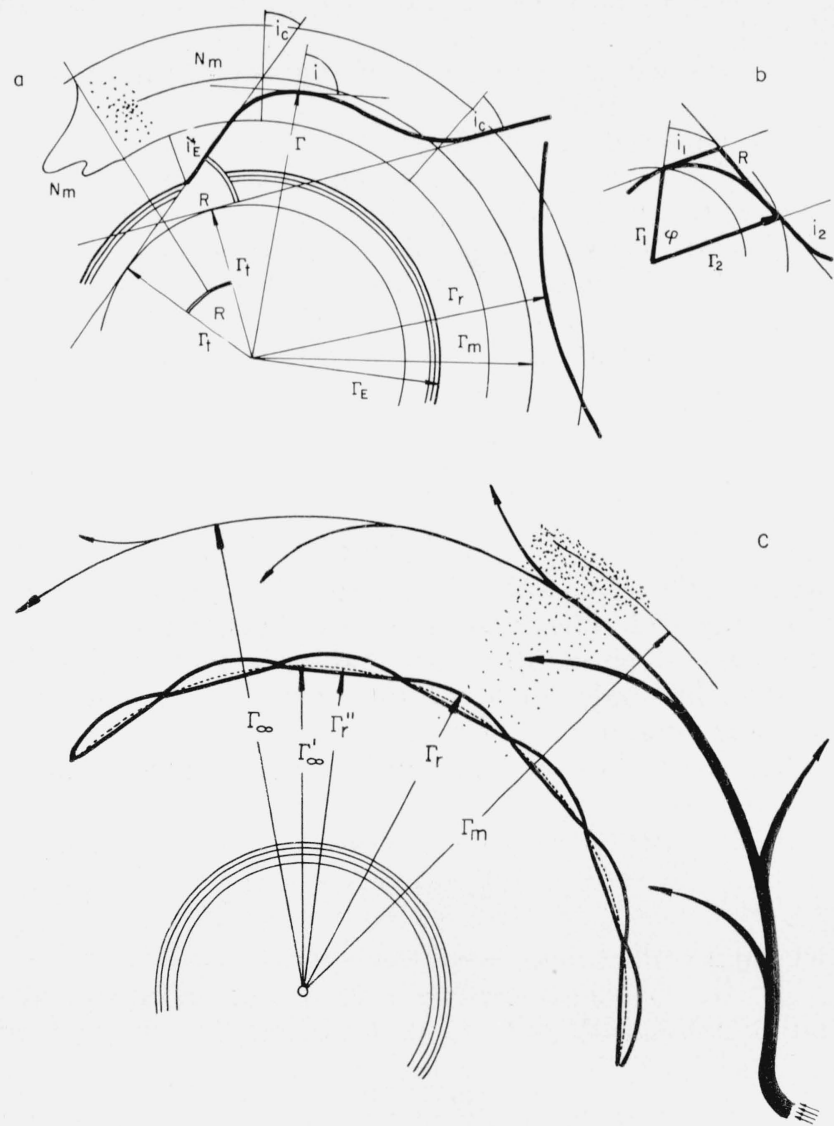

FigurE 1. Geometric representation of symbols used in the text. 
which is valid for any point on the ray path and the refraction formula

$$
d R=-\frac{d n}{d r} \frac{\tan i}{n} d r
$$

which expresses the deviation of a ray from its previous direction. The meaning of the symbols is best seen from figure la. $\left(r_{t}\right)$ is the sphere to which the ray path is tangent whenever it is outside the ionized layer $(N=0, n=1)$, while $\left(r_{t}^{(n)}\right)$ is the radius of the sphere to which the ray is tangent whenever it is within the ionized layer.

$$
r_{t}^{(n)} \frac{1}{n}=r_{t},
$$

where $(n)$ is the phase refractive index

$$
n=\sqrt{1-F \frac{N}{N_{m}}}, \quad F=\left(\frac{f_{c}}{f}\right)^{2}, f_{c}^{2} \propto N_{m},
$$

where $(f)$ is the wave frequency and $\left(f_{c}\right)$ is the critical or penetration frequency of the layer.

Three categories of radio propagation must be taken into account [Woyk, 1963]:

(a) waves penetrating the layer, [Woyk, 1958 and 1963]

(b) waves propagated along circular paths, [Chvojkova, 1954]

(c) reflected waves [Woyk, 1959].

(a) For waves penetrating the ionized layer, the following useful theorem has been derived [Chvojkova, 1958 and Woyk, 1963]. Both linear parts of a ray path-before entering the layer and after having left it - are merely turned about the center of curvature of the layer by the angle $(R)$, which can be expressed in the form

$$
R=\frac{F}{2} \frac{\sin i_{c}}{\cos ^{3} i_{c}} \frac{\int N d r}{N_{m} r_{m}},
$$

where $(R)$ is in radians. From this equation we see that angle of refraction of the ray penetrating a layer does not depend upon the shape of electron density profile, but only upon the total electron content in a vertical column of unit area.

This formula is accurate to $(q)$ decimal places only if

$$
\frac{F}{\cos ^{2} i_{c}} \leqslant 10^{-q} \text { and } \Delta \leqslant r_{m} 10^{-q},
$$

where $\Delta$ is the thickness of the layer.

(b) Radio waves can be propagated along two circular paths with radii $r_{\infty}$ and $r_{\infty}^{\prime}$ (fig. lc), defined by

$$
\frac{d n}{d r}=-\frac{n}{r} \text {. }
$$

The upper one $\left(r_{\infty}\right)$ lies just below the maximum of the layer $\left(r_{m}\right)$. Because of a strong dissipation of the beam propagated along level $\left(r_{\infty}\right)$, this type of (high ray) propagation is not of much practical use. The propagation along the lower one of radius $\left(r_{\infty}^{\prime}\right)$ lies just above lower boundary of the layer and is very stable. Rays which deviate slightly from this circular path are forced to return back to it perpetually either by reflection from the denser upper regions or because of the curvature of the layer. Thus the wave propagates alone $r_{\infty}^{\prime}$ in small oscillations.

(c) Formulas derived for reflected waves [Woyk, 1959] are either so complicated or so simplified that they are of no practical use. Therefore it has been necessary to search for a suitable electron-density distribution which would approximate the real one and which when introduced into the refraction formula would lead to an analytical solution.

\section{Analytical Ray Path Formulas}

Let us divide the ionosphere into zones as shown in figure 2. Each zone is bounded by spheres of radius $r_{x}$ or $r_{s}$. The $r_{x}$ 's denote levels of local maxima or minima of electron density, more specifically denoted as $r_{M}$ and $r_{\mu}$, respectively, and $r_{s}$ lies in the middle between the two extremes, such that

$$
r_{s}=\frac{1}{2}\left(r_{M}+r \mu\right)
$$

The actual electron density at a local maxima is given by $M$ and at a local minima by $\mu$, with $X$ representing the electron density at either extreme and that in the middle given by

$$
S=\frac{1}{2}(M+\mu) .
$$

The maxımum electron density of the entire ionosphere is further specified as $N_{m}$ and $r_{m}$ refers only to this level.

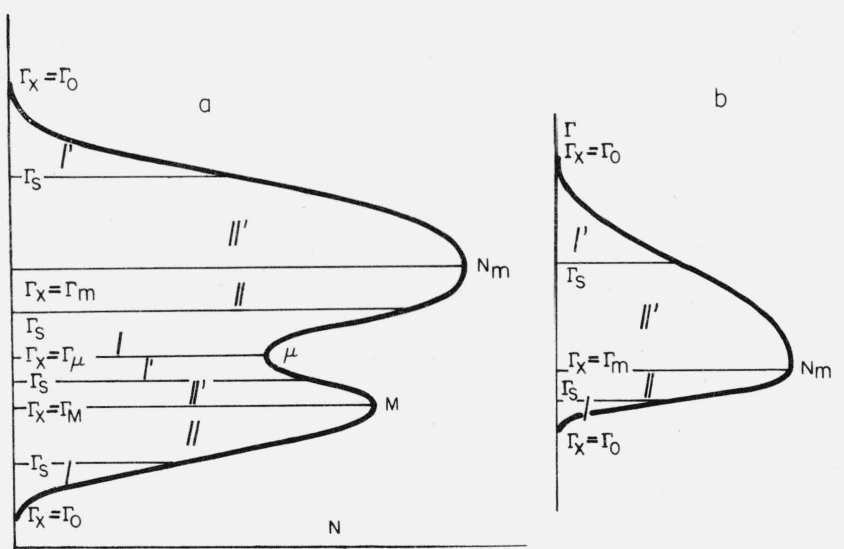


The shape of the electron density profile of each zone can be approximated by a quasi-parabola having this form:

$$
N=X+(S-X)\left(\frac{r_{s}}{r_{x}-r_{s}} \cdot \frac{r-r_{x}}{r}\right)^{2}
$$

The whole shape of the ionosphere can be represented by a series of such parabolas which all together give a smooth curve with the exception of the junctions at the levels $r_{s}$.

If we introduce (10) into (4) and make the following substitutions,

$$
\begin{gathered}
W=\frac{F(X-S)}{N_{m}-F X}\left(\frac{r_{s}}{r_{s}-r_{x}}\right)^{2}, \\
\bar{n}=\frac{n}{\sqrt{1-\frac{F X}{N_{m}}}},
\end{gathered}
$$

we have

$$
\bar{n}^{2}=1+W\left(\frac{r-r_{x}}{r}\right)^{2}
$$

and

$$
(\bar{n} r)^{2}=r^{2}(W+1)-2 W r_{x} r+W r_{x}^{2} .
$$

Consequently, it is also true that

$$
\bar{r}_{t}=\frac{r_{t}}{\sqrt{1-\frac{F X}{N_{m}}}} .
$$

With a simple nocturnal ionosphere (fig. 2b), it is appropriate to use

$$
N=\mp \frac{N_{m}}{2}\left[(1 \mp 1)-\left(\frac{r-r_{x}}{r} \cdot \frac{r_{s}}{r_{x}-r_{s}}\right)^{2}\right] .
$$

and therefore

$$
W=\mp \frac{F}{2 \mp(1 \mp 1) F}\left(\frac{r_{s}}{r_{s}-r_{x}}\right)^{2},
$$

where the upper (minus) signs refer to the outer (concave) zones I and I' and the lower (plus) signs refer to the inner (convex) zone II and II'.

Since we wish to derive an analytical formula, it has been found appropriate to introduce polar coordinates $r$ and $\varphi$. It follows from figure $1 \mathrm{~b}$ that

$$
\varphi=R-\left(i_{2}-i_{1}\right)
$$

and

$$
d \varphi=d R-d i
$$

To obtain an expression in terms of $r$ and $\varphi, d R$ and $d i$ must be introduced in (19) as functions of $r$ only. From Snell's Law we have

$$
\sin i=\frac{r_{t}}{n r}=\frac{\bar{r}_{t}}{\bar{n} r}
$$

and

$$
\bar{n}^{2} r^{2} \cos ^{2} i=(\bar{n} r)^{2}-\bar{r}_{t}^{2} .
$$

By differentiating (13), we get

$$
\frac{d \bar{n}}{d r}=\frac{W r_{x}\left(r-r_{x}\right)}{\bar{n} r^{3}},
$$

and substitution in (2),

$$
d R=-\bar{r}_{t} \frac{W r_{x}\left(r-r_{x}\right)}{r(\bar{n} r)^{2} \sqrt{(\vec{n} r)^{2}-\bar{r}_{t}^{2}}} d r .
$$

Rearranging (20) and differentiating, we have

$$
d i=\frac{d i}{d r} d i=-\bar{r}_{t} \frac{(W+1) r-W r_{x}}{(\bar{n} r)^{2} \sqrt{(\bar{n} r)^{2}-\bar{r}_{t}^{2}}} d r .
$$

Introducing (23) and (24) into (19) we get

$$
d \varphi=\bar{r}_{t} \frac{d r}{r \sqrt{r^{2}(W+1)-2 W r_{x} r+W r_{x}^{2}-\bar{r}_{t}^{2}}}
$$

which has an analytical solution. Depending upon initial conditions, there are three solutions that must be distinguished.

Case I. For $W<\left(\frac{\overline{r_{t}}}{r_{x}}\right)^{2}$, which is fulfilled in concave zones I and $I^{\prime}$, the solution of (25) is

$$
r=\frac{p}{1+\epsilon \sin k\left(\varphi-\varphi_{0}\right)},
$$

where

$$
p=\frac{W r_{x}^{2}-r_{t}^{2}}{W r_{x}}, \epsilon=\frac{\sqrt{(W+1) r_{t}^{2}-W r_{x}^{2}}}{W r_{x}}, k=\sqrt{1-\frac{W r_{x}^{2}}{r_{t}^{2}}} .
$$

Since the reference for the angle $\varphi$ has not as yet been chosen, let us set $\varphi_{0}=-90^{\circ}$ (at reflection $\varphi$ will be zero). Equation (26) can now be written as

$$
r=\frac{p}{1-\epsilon \cos k \varphi} \text {. }
$$

If $k=1$, the equation (28) is that of a simple ellipse. Usually $k$ is a large value and thus (28) is a quasiellipse whose $\varphi$ has been $k$-times contracted as indicated in figure 3a. For $\epsilon=0$ the path is circular and the wave propagated along $r_{\infty}$.

Case II. For $W>\left(\frac{\bar{r}_{t}}{r_{x}}\right)^{2}$, which is fulfilled in the con- 


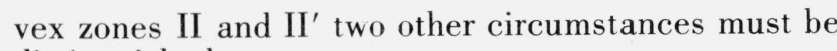
distinguished.

If $\epsilon^{2}<0$, the solution of (25) has the form

$$
r=\frac{p}{1-\epsilon^{\prime} \sinh k^{\prime} \varphi},
$$

where

$$
\epsilon^{\prime}=\sqrt{-\epsilon^{2}} \text { and } k^{\prime}=\sqrt{-k^{2}} .
$$

In this instance the ray passes the local layer without reflection as shown in figure $3 \mathrm{~b}$.

If $\epsilon^{2}>0$, the solution becomes

$$
r=\frac{p}{1-( \pm \epsilon) \cosh k^{\prime} \varphi}
$$

as represented in figure $3 \mathrm{c}$, and the wave is reflected. If we take $\epsilon$ positive, we are dealing with a wave coming from above the ionosphere and reflected outwards. A negative $\epsilon$ corresponds to waves from below reflected downwards. For $\epsilon=0$, the wave propagates along the circular path $r_{\infty}$.

Any ray path is sufficiently determined by $F, r_{x}$, $r_{s}$, and $r_{t}$. From these parameters one can find the values of $p, \epsilon$, and $k$, and hence one can determine all the partial curves of which the whole path is composed. Although composed of several fragments, the trajectory should be a smooth curve with a possible exception at $r_{s}$.

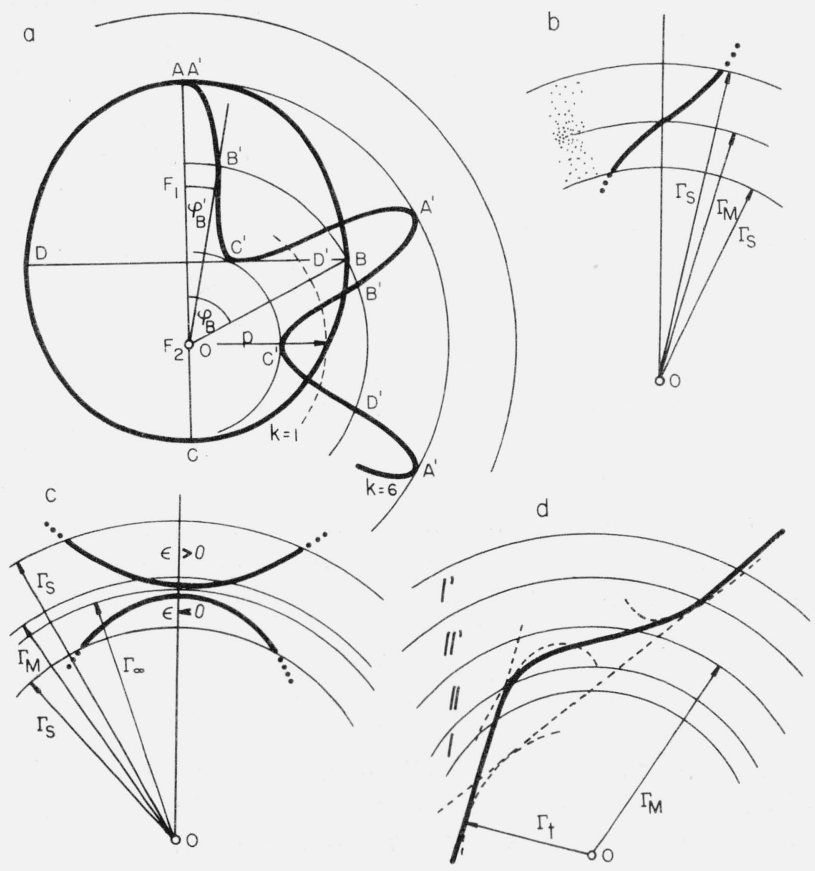

FIGURE 3. Representation of ray paths for the analytical solutions.
The value of $r_{t}$ must often be deduced from any one of several considerations: the angle of incidence at the earth's surface, the level of reflection, or the angle at a level $r$, or by the sphere $r_{t}^{(n)}$ to which the ray is tangential whenever it passes a level with the index of refraction $n$. In any of these cases, $r_{t}$ is easily determined from Snell's Law.

\section{Some Additional Remarks}

The above formulas can also be used when a magnetic field is present. If the space variation of the field is not great, it is sufficient to introduce the Appleton-Hartree formula into $W$.

The level of reflection $r_{r}$ is found from (28) and (31) by introducing $\varphi=0$. Thus

$$
r_{r}=\frac{p}{1 \pm \epsilon}
$$

No ray reflected below $r_{\infty}^{\prime}$ is reflected down. All are deviated into the denser upper regions, since they are less curved than the earth.

The circular paths are defined by $\epsilon=0$. The $r_{\infty}$ or $r_{\infty}^{\prime}$ are defined by $r=p$ or more specifically,

$$
r_{\infty} \text { or } r_{\infty}^{\prime}=\frac{W r_{x}}{W+1}
$$

It follows that one circular path $\left(r_{\infty}^{\prime}\right)$ lies in zones I only, just above the minimum electron density or lower boundary of the layer. The stability of this type of propagation is great as is evident from figures $1 \mathrm{c}$ and 3a. A deviation from the circular path brings the ray into oscillations along $r_{\infty}^{\prime}$, as previously mentioned. In zones I', no circular paths can occur and all rays reflected there are reflected outward.

The other circular path lies only in zone II, closely below the maximum of electron density. Figures lc and $3 \mathrm{c}$ show that these paths are unstable and the ray is easily dissipated on both sides.

With increasing $f,|W|$ decreases. $r_{\infty}$ and $r_{\infty}^{\prime}$ approach each other slowly, drifting slightly toward $r_{s}$. For thin layers, $\frac{\left|r_{s}-r_{x}\right|}{r_{x}}=10^{-3}$, they come together at $\frac{f}{f_{c}}=30$. For thicker layers, $\frac{\left|r_{s}-r_{x}\right|}{r_{x}}=2 \cdot 10^{-2}$, they join at $\frac{f}{f_{c}}=5$ (see table 1 ).

When a sharp gradient of electron density is to be investigated, formulas (28) and (31) or (29) can be used. For expressing $\frac{d N}{d t} \approx \infty$, however, we must set $r_{x}-r_{s}$ $\approx 0$, or $M$ and $\mu$ much above $N_{m}$ and much below $N=0$, respectively. This is consistent since the parts above $N_{m}$ and below $N=0$ are not investigated. When $\frac{d N}{d r}$ increases very much, $|W|$ and $\left|k^{2}\right|$ also increase very much. The oscillations of figure 3 a become more squeezed and the ray path is much sharper at the level of reflection. 
TABLE 1

\begin{tabular}{|c|c|c|c|c|c|}
\hline$f_{i} f_{c}$ & $3: 1$ & $5: 1$ & $7: 1$ & $10: 1$ & $100: 1$ \\
\hline$r_{s} / r_{m}$ & \multicolumn{5}{|c|}{$W$ in Zone $\mathrm{I}$} \\
\hline $\begin{array}{r}0.999 \\
.990 \\
.980\end{array}$ & $\begin{array}{r}-55,400 \\
-545 \\
-133\end{array}$ & $\begin{array}{r}-19,960 \\
-196 \\
-48\end{array}$ & $\begin{array}{r}-10,200 \\
-100 \\
-24.5\end{array}$ & $\begin{array}{r}-4,990 \\
-49 \\
-12\end{array}$ & $\begin{array}{r}-49.9 \\
-0.5 \\
-.12\end{array}$ \\
\hline$r_{s} / r_{m}$ & \multicolumn{5}{|c|}{$W$ in Zone II } \\
\hline $\begin{array}{r}0.999 \\
.990 \\
.980\end{array}$ & $\begin{array}{r}62,400 \\
612 \\
150\end{array}$ & $\begin{array}{r}20,800 \\
204 \\
50\end{array}$ & $\begin{array}{r}10,400 \\
102 \\
25\end{array}$ & $\begin{array}{r}5,050 \\
49.5 \\
12.1\end{array}$ & $\begin{array}{r}50 \\
0.49 \\
.12\end{array}$ \\
\hline$r_{s} / r_{m}$ & \multicolumn{5}{|c|}{$W$ in Zone II' } \\
\hline $\begin{array}{l}1.001 \\
1.010 \\
1.020\end{array}$ & $\begin{array}{r}62,500 \\
638 \\
162\end{array}$ & $\begin{array}{r}20,800 \\
212 \\
54\end{array}$ & $\begin{array}{r}10,100 \\
106 \\
27\end{array}$ & $\begin{array}{r}5,050 \\
51.5 \\
13.1\end{array}$ & $\begin{array}{r}50 \\
0.51 \\
.13\end{array}$ \\
\hline$r_{s} / r_{m}$ & \multicolumn{5}{|c|}{$W$ in Zone $\mathrm{I}^{\prime}$} \\
\hline $\begin{array}{l}1.001 \\
1.010 \\
1.020\end{array}$ & $\begin{array}{r}-55,600 \\
-567 \\
-144\end{array}$ & $\begin{array}{r}-20,000 \\
-204 \\
-52\end{array}$ & $\begin{array}{r}-10,200 \\
-104 \\
-26.5\end{array}$ & $\begin{array}{r}-5,010 \\
-51 \\
-13\end{array}$ & $\begin{array}{r}-50 \\
-0.51 \\
-.13\end{array}$ \\
\hline$r_{s} / r_{m}$ & \multicolumn{5}{|c|}{$r_{\infty}^{\prime} / r_{m}$ in Zone I } \\
\hline $\begin{array}{r}0.999 \\
.990 \\
.980\end{array}$ & $\begin{array}{r}0.998 \\
.982 \\
.967\end{array}$ & $\begin{array}{r}0.998 \\
.985 \\
.980\end{array}$ & $\begin{array}{c}0.9981 \\
.990 \\
\ldots \ldots \ldots \ldots\end{array}$ & $\begin{array}{c}0.9982 \\
\ldots \ldots \ldots \ldots \ldots \\
\ldots \ldots \ldots \ldots \ldots . . . . . . \\
\end{array}$ & \\
\hline$r_{s} / r_{m}$ & \multicolumn{5}{|c|}{$r_{\infty} / r_{m}$ in Zone II } \\
\hline $\begin{array}{r}0.999 \\
.990 \\
.980\end{array}$ & $\begin{array}{c}1.0000 \\
0.998 \\
.993\end{array}$ & $\begin{array}{c}1.0000 \\
0.995 \\
.980\end{array}$ & $\begin{array}{c}0.9999 \\
.990 \\
\ldots \ldots \ldots\end{array}$ & $\begin{array}{c}0.9998 \\
\ldots \ldots \ldots \ldots \ldots \\
\ldots \ldots \ldots \ldots \ldots \ldots\end{array}$ & \\
\hline$r_{x}$ & \multicolumn{5}{|c|}{$W$ in Zones II or II' } \\
\hline 0 & 0.0625 & 0.0208 & 0.0104 & 0.00505 & 0.00005 \\
\hline$r_{x}$ & \multicolumn{5}{|c|}{$W$ in Zones $\mathrm{I}$ or $\mathrm{I}^{\prime}$} \\
\hline 0 & -0.0555 & -0.0200 & -0.0102 & -0.00500 & -0.00005 \\
\hline
\end{tabular}

Some apparent discrepancies arising during an analysis are usually avoided if we consider that we wish to investigate only the regions bounded by $r_{x}$ and $r_{s}$. Outside this region, the theoretical curves increase rapidly above $N_{m}$ or below $N=0$, and the region I goes over into $\mathrm{I}^{\prime}$ at the level $r_{x}$. Thus outside the boundaries $r_{x}$ and $r_{s}$, the result no longer represents the investigated case.

At $W \approx 0$ attention must be paid to the fact that in the zones II and $\mathrm{II}^{\prime}(28)$ is valid even for positive $W<\left(\frac{\bar{r}_{t}}{r_{x}}\right)^{2}$ while (29) and (31) are true only for $W>\left(\frac{\overline{r_{t}}}{r_{x}}\right)^{2}$.
This condition exists only when $f \gg f_{c}$ or when one of the boundary levels approaches $r_{x}=0$. Since this eventuality is of no practical importance for terrestrial radio communication it will be analyzed elsewhere [Woyk, 1965].

It is surprising that in a zone bounded by $r_{x}=0$, the ray path is rectilinear. This is because (10) gives a constant electron density $N=\frac{N_{m}}{2}$ in the entire zone, except where $r=0$.

For the sake of a rapid survey, some values of $W$ and $r_{\infty}$ have been given in table 1 . They correspond to a simple layer similar to figure $2 \mathrm{~b}$ but symmetrical about $r_{m}$. For $r_{x}=0$, the values of $W$ refer to $(k)$; they are independent of $r_{s}$. When $r_{x} \approx 0$, (7) corresponds to a layer much different from that in figure $2 \mathrm{~b}$.

I express my gratitude to Dr. K. Davies and Dr. Dieminger for the invitation to attend the Lindau meeting and for suggesting the investigation presented in this paper. Further I thank Dr. K. Davies, Dr. R. G. Maliphant, and the scientific staff of the Kiruna Observatory for many important suggestions leading to a rapid solution of the given problem.

\section{References}

Chvojkova, E. (E. Woyk) (1954), Ueber den Weltumlauf der Radiostrahlen (Refraction der Kurzwellen in nichtparabolischer Schich), Bull. Astr. Insts. Csl 5, 104 and 110.

Chvojkova, E. (1958), Propagation of radio waves from cosmical sources, Nature 181, 105.

IQSY Notes (Sept. 1963), Report on the Lindau meeting on oblique propagation.

Woyk-Chvojkova, E. (1958), Determination of radio propagation elements due to an artificial earth satellite, Nature 181, 11951196.

Woyk, E. (E. Chvojkova) (1959), The refraction of radio waves by a spherical ionized layer, J. Atmospheric Terrest. Phys. 16, 124135.

Woyk, E. (1959), The antipodal reception of Sputnik III, Proc. Inst. Rad. Engin. 47, 1144.

Woyk, E. (1961), Antipodal reception of satellite signals and telecommunication on small planets, Bull. Astr. Insts. Csl. 12, 1.

Woyk, E. (1963), Radio communication in astronautics (from the geometrical point of view), Publication 49 of the Astronom. Instit. Czechosl.

Woyk, E. (1964), Radiophysics and on analytical formula for radio path determination, Nature 201, 804-806.

Woyk, E. (1965), On the apparent coincidence between geometrical optics and celestial mechanics, Bull Astr. Insts. 16 (in press), and Radio Path formulae for radioastronomy and astronautics, ibid. (in press)

(Paper 69D3-483) 